\section{Arabidopsis cmt3 chromomethylase mutations block non-CG methylation and silencing of an endogenous gene}

\author{
Lisa Bartee, Fabienne Malagnac, \\ and Judith Bender ${ }^{1}$ \\ Department of Biochemistry and Molecular Biology, \\ Bloomberg School of Public Health, Johns Hopkins \\ University, Baltimore, Maryland 21205, USA
}

\begin{abstract}
Plants maintain cytosine methylation at CG and nonCG residues to control gene expression and genome stability. In a screen for Arabidopsis mutants that alter methylation and silencing of a densely methylated endogenous reporter gene, we recovered 11 loss-of-function alleles in the CMT3 chromomethylase gene. The cmt3 mutants displayed enhanced expression and reduced methylation of the reporter, particularly at non-CG cytosines. CNG methylation was also reduced at repetitive centromeric sequences. Thus, CMT3 is a key determinant for non-CG methylation. The lack of CMT homologs in animal genomes could account for the observation that in contrast to plants, animals maintain primarily CG methylation.
\end{abstract}

Received April 23, 2001; revised version accepted May 22, 2001.

In many eukaryotes, including mammals, higher plants, and some species of fungi, cytosine methylation plays an important role in genome stability and development by altering chromatin structure and patterns of gene expression. In mammalian genomes, methylation is found primarily at cytosines in the symmetric context $5^{\prime}$-CG-3' (CG), whereas in plant and fungal genomes methylation is found on both CG and non-CG residues /Yoder et al. 1997; Colot and Rossignol 1999; Finnegan and Kovac 2000). Mammals and higher plants carry related cytosine methyltransferases of the Dnmt1/MET1 class that have been implicated by mutational analysis as enzymes that maintain the bulk of genomic methylation ( $\mathrm{Li}$ et al. 1992; Finnegan et al. 1996; Ronemus et al. 1996). Another class of chromomethylases (CMTs) has been identified by analysis of Arabidopsis thaliana genomic sequences (Henikoff and Comai 1998; McCallum et al. 2000). The CMT class is characterized by the presence of a chromodomain amino acid motif between the cytosine methyltransferase catalytic motifs I and IV. There are three CMT genes encoded in Arabidopsis: CMT1, CMT2,

[Key Words: Cytosine methyltransferase; gene silencing; Arabidopsis thaliana]

${ }^{1}$ Corresponding author.

E-MAIL jbender@welchlink.welch.jhu.edu; FAX (410) 955-2926.

Article and publication are at http://www.genesdev.org/cgi/doi/10.1101/ gad.905701. and CMT3 (Henikoff and Comai 1998; Finnegan and Kovac 2000; McCallum et al. 2000). In the Wassilewskija (WS) strain background used for this study, CMT2 and CMT3 are predicted to encode functional proteins, whereas the CMT1 coding sequence is disrupted by an Eve1 (Henikoff and Comai 1998) retroelement insertion (J. Bender, unpubl.). CMT genes have also been identified in several other plant species including Brassica and maize, but not in fungal or animal systems (Rose et al. 1998; Finnegan and Kovac 2000). Recently, Arabidopsis CMT3 (Lindroth et al. 2001) and the maize CMT homolog ZMET2 (Papa et al. 2001) have been implicated in the maintenance of CNG methylation.

In the genome of Arabidopsis, duplicated genes encoding the tryptophan pathway enzyme phosphoribosylanthranilate isomerase (PAI) provide a well-characterized example of endogenous genes that are densely methylated with both CG and non-CG methylation (Luff et al. 1999). In the Arabidopsis strain WS, there are four methylated $P A I$ genes at three unlinked loci: a singlet $P A I 2$ gene that encodes functional enzyme, a singlet $P A I 3$ gene that does not encode functional enzyme, and a tailto-tail inverted repeat of the PAI1 and PAI4 genes (PAI1$P A I 4)$ in which the PAI1 gene encodes functional enzyme and the PAI4 gene does not (Bender and Fink 1995; Melquist et al. 1999). The functional singlet $P A I 2$ gene is silenced by methylation (Bender and Fink 1995; Jeddeloh et al. 1998; Melquist et al. 1999). In contrast, the functional PAI1 gene in the inverted repeat is expressed despite dense methylation in the body of the gene, providing sufficient PAI enzyme for a wild-type plant phenotype (Melquist et al. 1999). It is likely that the WS PAI1 gene eludes silencing by methylation because of novel promoter sequences lying upstream of the methylated region (Melquist et al. 1999; J. Bender, unpubl.).

Here we describe the isolation and characterization of mutations in the CMT3 chromomethylase gene from a genetic screen for reduced PAI methylation. Southern blot analysis and bisulfite genomic methylation sequencing indicate that $c m t 3$ mutations confer a partial loss of CG methylation and a strong loss of non-CG methylation (both CNG and asymmetric cytosines) from the PAI genes. Southern blot analysis of repetitive methylated genomic sequences indicates that $c m t 3$ mutations also confer reduced CNG methylation on these regions. In contrast to characterized Arabidopsis mutations that confer globally decreased methylation (Finnegan et al. 1996; Kakutani et al. 1996; Ronemus et al. 1996), the cmt3 mutations do not lead to pleiotropic effects upon inbreeding, suggesting that CMT3 function is specialized for only a subset of methylated regions in the genome.

\section{Results and Discussion}

To identify factors that control methylation and silencing of the WS PAI genes, we isolated a mutant variant of WS, pai1C251Y, in which silencing of the methylated singlet PAI2 gene can be visualized by the intensity of a blue fluorescent plant phenotype under ultra-violet (UV) light (Bartee and Bender 2001). In the pai1C251Y strain, the only potential sources of PAI enzyme activity are the PAI1 gene, which is crippled by a missense mutation, 
A

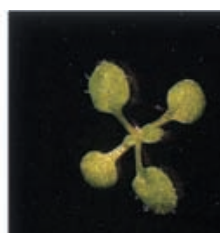

WS

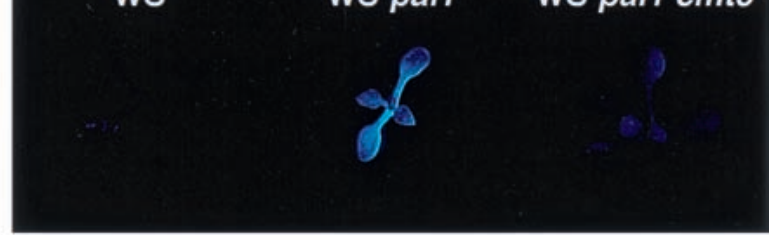

B

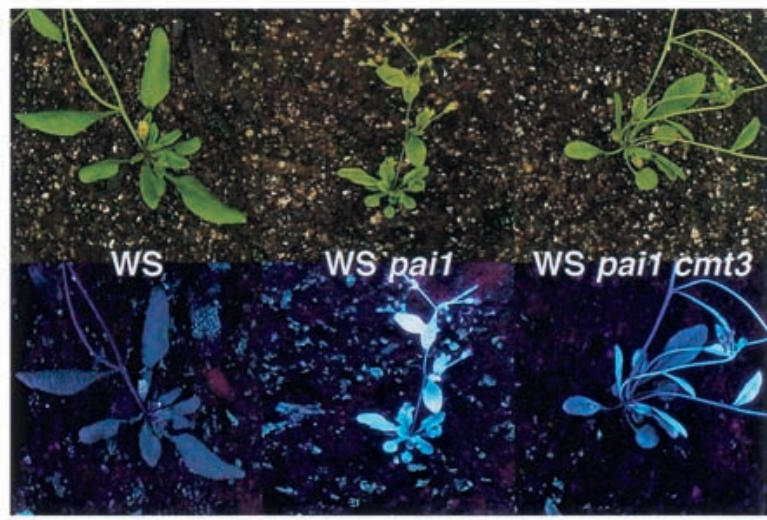

C

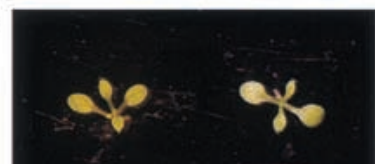

WS pai1 cmt3 (CMT3)

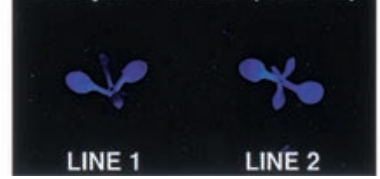

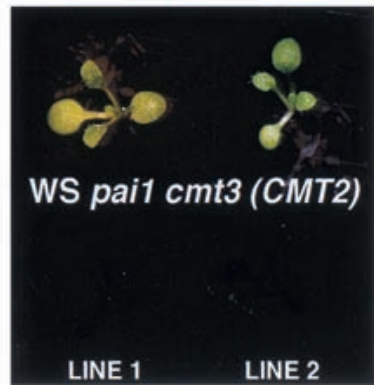

LINE 1

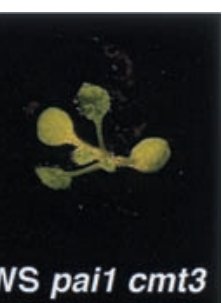

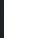

\section{9}

From this screening strategy we isolated 11 loss-offunction alleles in the CMT3 gene (see below and Materials and Methods). The cmt3 mutants in the pai1C251Y background displayed strongly reduced fluorescence in early seedling development and partially reduced fluorescence in adult plants, with increased size, decreased bushiness, and increased fertility (Fig. 1). These intermediate fluorescent $\mathrm{cmt} 3$ isolates did not revert to nonfluorescence, which is diagnostic of loss of residual PAI2 methylation (Bender and Fink 1995), at a detectable frequency. They displayed partially increased cleavage with HpaII and strongly increased cleavage with $M s p I$ for the PAI genes relative to parental pai1C251Y (Fig. 2A). The cleavage pattern suggested that the $c m t 3$ mutants were

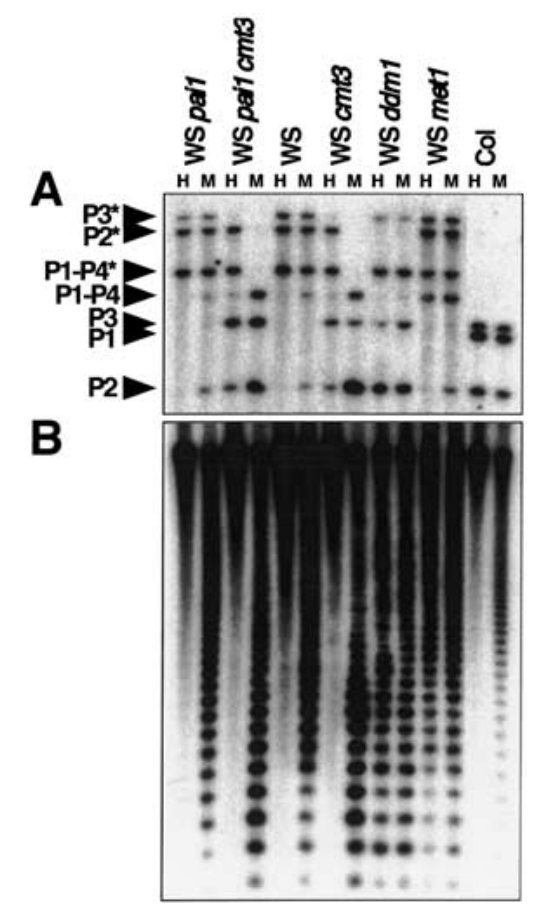

Figure 2. cmt3 mutations confer reduced $P A I$ and $C E N$ methylation. (A) Genomic DNAs prepared from 4-week-old plants of the indicated genotypes were cleaved with either $H p a I I(H)$ or $\operatorname{MspI}(\mathrm{M})$ and used for Southern blot analysis with a $P A I$ probe (Bender and Fink 1995). (P1-P4) PAI1-PAI4; (P2) PAI2; (P3) PAI3; (P1) Columbia (Col) strain PAI1; asterisks indicate the positions of species methylated at internal HpaII/MspI sites (Bender and Fink 1995; Luff et al. 1999). The Col strain is included as a control for the positions of unmethylated PAI2 and PAI3 species. $(B)$ The blot shown in $A$ was reprobed with a $180-b p$ CEN repeat probe. The phenotypes of the cmt3G456D allele shown are representative of the phenotypes observed with 10 other $c m t 3$ alleles. 
most affected in maintenance of CNG methylation of the PAI genes. To determine whether the cmt3 mutants also affected methylation of a highly repeated genomic sequence, we reprobed the HpaII/MspI Southern blot with a probe to the 180-bp centromere-associated repeat (CEN) sequences (Vongs et al. 1993). This probe revealed little effect on HpaII cleavage but increased MspI cleavage, consistent with the pattern observed for the $P A I$ genes (Fig. 2B). A similar pattern of increased MspI cleavage was also observed at the repeated $r D N A$ (data not shown). All of $11 \mathrm{cmt} 3$ alleles tested had identical methylation patterns in these assays. Moreover, when the cmt3 alleles were segregated away from the pai1C251Y allele into a wild-type WS background, they also displayed identical methylation patterns in these assays (Fig. 2). The PAI and CEN methylation patterns were distinct from the patterns induced by the characterized $d d m 1$ and met1 methylation-deficient mutations (Fig. 2; Bartee and Bender 2001).

To more precisely determine methylation patterns in the $c m t 3$ mutant background, we performed genomic sequencing of methylation patterns in the PAI1 and PAI2 promoter regions of a representative $c m t 3$ allele by using sodium bisulfite mutagenesis (Frommer et al. 1992). This analysis revealed that the majority of methylated cytosines $(87 \%$ in PAI1 and $70 \%$ in PAI2) occurred at CG residues (Fig. 3; Table 1). Compared with the wildtype WS PAI1 promoter (Luff et al. 1999; Table 1), CG methylation was reduced $34 \%$, CNG methylation was eliminated, and asymmetric methylation was reduced $93 \%$; in the PAI2 promoter, CG methylation was reduced $8 \%$, CNG methylation was reduced $92 \%$, and non-CG methylation was reduced $75 \%$. Thus, loss of CMT3 function has a strong effect on maintenance of
Table 1. Effects of a cmt3 mutation on patterns of PAI promoter cytosine methylation ${ }^{a}$

\begin{tabular}{llcccc}
\hline \multirow{2}{*}{ Strain } & $\begin{array}{l}\text { PAI } \\
\text { gene }\end{array}$ & \multicolumn{1}{c}{ CG } & CNG & Other C & Total C \\
\hline WS & $P A I 1$ & $115(100 \%)$ & $61(100 \%)$ & $149(100 \%)$ & $325(100 \%)$ \\
$c m t 3^{b}$ & $P A I 1$ & $76(66 \%)$ & $0(0 \%)$ & $11(7 \%)$ & $87(27 \%)$ \\
WS & $P A I 2$ & $122(100 \%)$ & $53(100 \%)$ & $184(100 \%)$ & $359(100 \%)$ \\
$c m t 3$ & $P A I 2$ & $112(92 \%)$ & $4(8 \%)$ & $45(25 \%)$ & $161(45 \%)$ \\
\hline
\end{tabular}

${ }^{a}$ The numbers of methylated cytosines in the indicated sequence contexts for eight independent top strand bisulfite sequencing clones for the indicated $P A I$ promoter regions of the indicated strains are shown. The wild-type Wassilewskija (WS) data are derived from data published previously in Luff et al. (1999). The cmt3 data are from this work, and are shown in diagram form in Figure 3.

${ }^{\mathrm{b}}$ The sequenced DNA was from the WS pai1C251Y cmt3G456D strain.

CNG and asymmetric methylation and a weaker effect on maintenance of CG methylation. These results are consistent with reports that Arabidopsis CMT3 and maize ZMET2 are important for maintenance of CNG methylation at various genomic sites (Lindroth et al. 2001; Papa et al. 2001), but they further show that CMT3 is also important for maintenance of asymmetric methylation for the $P A I$ genes. This result implies either that CMT3 directly controls both symmetric and asymmetric methylation or that the reduction in symmetric methylation in the cmt3 mutant background causes reduced asymmetric methylation as a secondary consequence. Because the methylated sequences in the promoter and first exon of the PAI2 reporter gene $(\sim 370 \mathrm{bp})$ contain only 16 dispersed CG motifs, loss of non-CG methylation significantly hypomethylates this region of the gene (Fig. 3), ac-

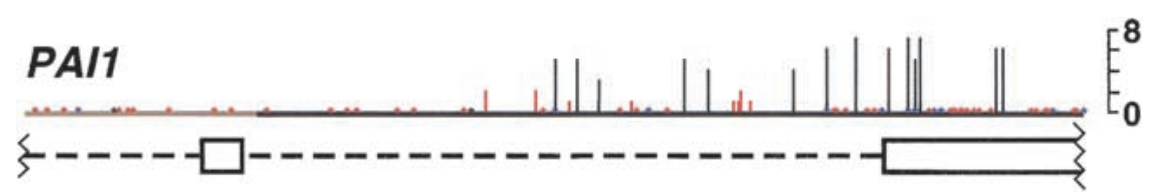
counting for enhanced PAI2 expression in the suppressor mutant.

The cmt3 mutant locus in the pai1C251Y cmt3 suppressor isolates was mapped by crosses with the polymorphic strain $\mathrm{Nd}-0$, which has a similar arrangement of densely methylated PAI genes as found in WS (Melquist et al. 1999). $F_{2}$ progeny with weakly fluorescent phenotypes diagnostic of homozygosity for both pai1C251Y and cmt3 were identified by visual inspection under UV light and confirmed by MspI Southern blot for strong PAI cleavage similar to that observed in the parental suppressor isolates. A mapping population of $\mathrm{F}_{2}$ plants that fulfilled these criteria was then used to score for genomic loci linked to the suppressed phenotype. The mapping analysis revealed linkage to a single locus on the lower arm of chromosome 1. Because the CMT3 putative cytosine methyltransferase 
gene maps to this locus, we focused on this gene as a candidate. Within each mapping population, we found complete linkage to a polymorphic marker that lies within $100 \mathrm{~kb}$ of the CMT3 gene. To confirm that the CMT3 gene was in fact the site of the methylation suppressor mutations, we cloned and sequenced the gene from the 11 mutant isolates. Sequencing revealed a single base change in the CMT3 coding sequence in each isolate. Three of the mutant alleles affected absolutely conserved amino acids in the methyltransferase catalytic domain, including the representative cmt3G456D allele used for bisulfite sequencing. Another allele was predicted to prematurely terminate the protein. Two alleles created splice junction mutations. The remaining five alleles affected amino acids between methyltransferase motif IV and the C terminus of the protein that are highly conserved among the CMT genes (Fig. 4).

To further confirm that the CMT3 gene was the mutant locus, we transformed the pai1C251Y cmt3 isolates with a wild-type WS genomic clone of the CMT3 gene. Transformant seedlings were strongly fluorescent, similarly to those of the pai1C251Y strain (Fig. 1). Transformant lines assayed by Southern blot analysis in the T2 generation showed remethylation of the PAI2 gene to the levels observed in the original pai1C251Y strain (data not shown). Thus, the cloned CMT3 gene could complement the mutant methylation defects. As a control, the representative pai1C251Y cmt3G456D mutant was also transformed with a wild-type WS genomic clone of the CMT2 gene. CMT2 transformant seedlings were weakly fluorescent, similarly to those of the untransformed parental strain (Fig. 1), and did not display detectable remethylation of PAI2. This analysis shows that CMT2 cannot substitute for CMT3 function. In this regard, it is interesting to note that CMT2 differs from CMT3 primarily in its $\mathrm{N}$ terminal sequence (Fig. 4).

Previously characterized methylation-deficient Arabidopsis strains with defects in either the SWI2/SNF2 chromatin remodeling factor-related gene DDM1 (Jeddeloh et al. 1999) or the Dnmt1-related MET1 cytosine methyltransferase gene display progressive developmental abnormalities (Finnegan et al. 1996; Kakutani et al. 1996; Ronemus et al. 1996). Our preliminary analysis of six-generation-inbred pai1C251Y cmt3 and two-generation-inbred $\mathrm{cm} t 3$ strains revealed no obvious segregation of morphological changes. This difference between cmt3 and other methylation-deficient mutants is likely to reflect the fact that CG methylation is retained to a higher degree in $c m t 3$ than in ddm1 or met 1 (Fig. 2; Bartee and Bender 2001). Because many of the endogenous methylated sites in the Arabidopsis genome, such as the CEN repeats (Fig. 2; Vongs et al. 1993; Lindroth et al. 2001), and the promoter of the FWA homeo-domain gene (Soppe et al. 2000; Lindroth et al. 2001), carry primarily CG methylation, cmt3 mutations would not be expected to strongly affect these loci. Instead, CMT3 most likely acts as a reinforcing methylase that adds an extra layer of methylation to particular genomic regions such as the $P A I$ genes, in which the in-

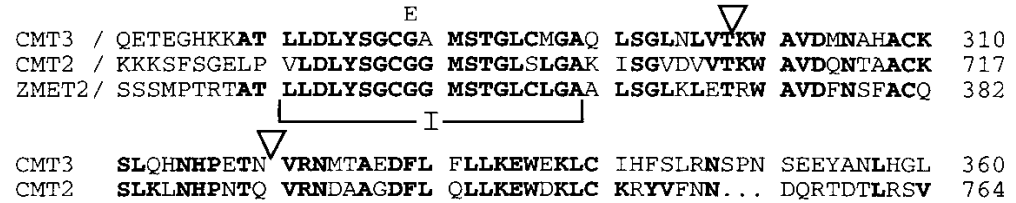

CMT2 SLKLNHPNTO VRNDAAGDFL OLLKEWDKLC KRYVFNN .. DORTDTLRSV 764 ZMET2 SLKYNHPQTE VRNEKADEFL ALLKEWAVLC KKYVQD ............ . . 419

CMT3 NNVEDNEDVS EESENEDDGE VFTVDKIVGI SFGVPKKLLK RGLYLKVRWL 410 CMT2 NSTKETSESS SSSDDDSDSE EYEVEKLVDI CFGDPDKTGK NGLKFKVHWK 814 ZMET2 DSNLASSEDQ ADEDSPLDKD EFVVEKLVGI CYGGSDR...E NGIYFKVQWE 467 CMT3 NYDDSHDTWE PIEGLSNCRG KIEEFVKLGY KSGILPLPGG VDVVCGGPPC 460 CMT2 GYRSDEDTWE LAEELSNCOD AIREFVTSGF KDKILPLPGR VGVICGGPPC 864 ZMET2 GYGPEEDTWE PIDNLSDCPQ KIREFVQEGH KRKILPLPGD VDVICGGPPC 517 D chromodomain-

CMT3 QGISGHNRFR NLLDPLEDQK NKQLLVYMNI VEYLKPKFVL MENVVDMLKM 510 CMT2 QGISGYNRHR NVDSPLNDER NQQIIVFMDI VEYLKPSYVL MENVVDILRM 914 ZMET2 QGISGFNRYR NRDEPLKDEK NKQMVTFMDI VAYLKPKYVL MENVVDILKF 567 - IV F

CM'3 AKGYLARFAV GRLLOMNYQV RNGMMAAGAY GLAQFRLRFF LWGALPSEII 560 CMT2 DKGSIGRYAL SRLVNMRYOA RLGIMTAGCY GLSOFRSRVF MWGAVPIKNI 964 ZMET2 ADGYLGKYAL SCLVAMKYQA RLGMMVAGCY GLPQFRMRVF LWGALSSMVL 617 CMT3 PQFPLPTHDL VHRGNIVKEF OGNIVAYDEG HTVKLADKLL LKDVISDLPA CMT2 PPFPLPTHDV IVRYGLPLEF ERNVVAYAEG QPRKLEKALV LKDAISDLPH 1014 ZMET2 PKYPIPTYDV VVRGGAPNAF SOCMVAYDET QKPSIKKALL LGDAISDLPK 667

CMT3 VANSEKRDEI TYDKDPTTPF QKFIRLRKDE ASGSQSKSKS KKHV..LYDH 658 CMT2 VSNDEDREKL PYESLPKTDF QRYIRSTKRD LTGSAIDNCN KRTML.LHDH 1063 ZMET2 VQNHQPNDVM EYGGSPKTEF QRYIRLSRKD MLDWSFGEGA GPDEGKLLDH 717 $\nabla_{\text {GANFRDE }}^{\mathrm{K}}$

CMT3 HPLNLNINDY ERVCQVPKRK GANFRDFPGV IVGPGNVVKL EEGKERVKLE 708 CMT2 RPFHINEDDY ARVCQIPNRK GANFRDLPGI IV.RNNTVCR DPSMEPVILP 1112 ZMET2 QRLRLNNDDY ERVQQIPVKK GANFRDLKGV RVGANNIVEN DPEIERVKLS 767

CMT3 SGKTLVPDYA LTYVGKSCK PFGRLWWEI VPTVVTRAET HNOVIIHPEQ 758 CMT2 SGKPLVPGYV FTFOOGKSKR PFARLWWDET VPTVLTVPTC HSOALLFPEO 1162

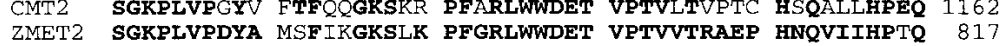

CMT2 NRVLSIRENA RLQGFPDDYK LFGPPKQKYI QVGNAVAVPV AKALGYALGT 808 CMT3 DRVLTIRESA RLQGFPDYFQ FCGTIKERYC OIGNAVAVSV SRALGYSLGM 1212 ZMET2 ARVLTIRENA RLQGFPDYYR LFGPIKEKYI QVGNAVAVPV ARALGYCLGQ 867 CMT3 AFQGLAVGKD PLLTLPEGFA FMKPTLPSEL A* CMT2 AFRGLA. RDE HLTKLPQNFS HSTYPQLQET IPH* ZMET2 AYLGESEGSD PLYOLPPSFT SVGGRTAGQA RASPVGTPAG EVVEQ*

Figure 4. Positions of mutations in CMT3. The predicted amino acid sequences of Wassilewskiya (WS) CMT3, WS CMT2, and maize ZMET2 are shown aligned along their conserved $\mathrm{C}$-terminal regions. The $\mathrm{N}$ termini, upstream of the backslash at the beginning of each sequence, are unrelated. CMT3 introns are indicated by inverted triangles above the sequence. CMT3 missense mutations are indicated above the affected residues. The stop mutation is indicated by an asterisk, and the splice donor and acceptor site mutations are indicated by an $\mathrm{x}$ to the left or right, respectively, above the affected introns. Residues identical between proteins are highlighted in boldface. Conserved sequence motifs are indicated under the alignment. GenBank accession nos. are: AF383170 for WS CMT3 and AF383171 for WS CMT2. 
creased methylation density leads to increased silencing. A specific model is that the basal layer of CG methylation provided by other functions such as MET1 could serve as a guide for CMT3, which would then decorate the basal layer with extra CG and non-CG methylation. CMT3 recruitment to targeted regions could involve chromatin protein interactions with the chromodomain motif (Henikoff and Comai 1998), along with interactions mediated by the unique $\mathrm{N}$-terminal sequences.

Because fungi such as Neurospora crassa and Ascobolus immersus can maintain non-CG methylation (Selker et al. 1993; Goyon et al. 1994), these organisms might encode CMT genes. Conversely, because animals such as humans and mice lack non-CG methylation, these organisms are predicted to lack CMT genes, as is the case from analyses of current sequence databases. The apparent lack of CMT-like methylases in animal genomes (Finnegan and Kovac 2000) suggests that animals have evolved alternate mechanisms for reinforcing chromatin states.

\section{Materials and methods}

Mutant isolation and sequencing

Seeds of WS pai1C251Y were mutagenized with ethylmethane sulfonate (Niyogi et al. 1993) and grown up as 20 pools of 500 M1 plants each; M2 progeny seeds were collected from each pool. Approximately 1000 seedlings from each M2 pool were grown on agar medium for 2 wk and then screened with a hand-held short wavelength UV light source for individuals with reduced fluorescence. Putative mutants were transplanted to soil, and genomic DNA prepared from a single leaf was used for Southern blot analysis of methylation patterns. From the screen of 20,000 M2 seedlings, all 11 isolates that displayed cmt3 methylation patterns (Fig. 2) proved to be $c m t 3$ alleles by mapping, sequencing, and complementation analysis. Ten other isolates with distinct methylation patterns as determined by HpaII/MspI Southern blot analysis were also recovered, but these isolates remain to be characterized. Interestingly, none of the other isolates displayed the $P A I$ and CEN Southern blot phenotypes diagnostic of $d d m 1$ mutations in the WS background (Fig. 2). Because we expect to recover $d d m 1$ alleles from the screen (Bartee and Bender 2001), this observation suggests that the screen is not yet saturated.

The cmt3 mutant locus was mapped to the lower arm of chromosome 1 with standard CAPS (Konieczny and Ausubel 1993) and simple sequence length polymorphism (SSLP) (Bell and Ecker 1994) markers that are polymorphic between WS and Nd-0. It was further localized between the markers NF5I14 and NF22K20 (http://www.arabidopsis.org/servlets/ mapper). Linkage to CMT3 was determined with the T17F3 marker, which lies within $100 \mathrm{~kb}$ of the CMT3 gene: forward primer $5^{\prime}$-gacataataccgagtacccac-3'; reverse primer $5^{\prime}$-ccaccaccttgcactgccgacc- $3^{\prime}$; in WS a 354-bp product cleaves once with MspI into $240 \mathrm{bp}$ and $114 \mathrm{bp}$ fragments, whereas the Nd-0 product is uncleaved.

Mutant alleles of CMT3 were amplified by PCR from genomic DNA. Products from two independent PCRs were cloned and sequenced for each allele. Alleles that changed restriction sites (both splice site mutations, cmt3G456D, cmt3G465D, and cmt3R703K) were confirmed by PCR amplification of the mutant region followed by cleavage with the appropriate enzyme.

\section{CMT genomic and $c D N A$ clones}

The CMT3 transgene is a WS genomic fragment extending from $2.9 \mathrm{~kb}$ upstream of the start codon to $0.8 \mathrm{~kb}$ downstream of the stop codon subcloned into the pBIN19 transformation vector (Bevan 1984). The CMT2 transgene is a WS genomic fragment extending from $1.7 \mathrm{~kb}$ upstream of the start codon to $0.9 \mathrm{~kb}$ downstream of the stop codon subcloned into pBIN19. Both clones were isolated by hybridization from a WS genomic library (Bender and Fink 1995). Both clones were sequenced across the coding region to confirm their structure and determine WS polymorphisms. Transgenes were introduced into the WS pai1C251Y cmt3G456D strain by an in planta transformation method (Clough and Bent 1998).
The CMT3 predicted amino acid sequence was determined by cloning and sequencing a cDNA isolate generated by RT-PCR from WS wholeplant RNA. CMT2 and ZMET2 amino acid sequences are predicted from the WS CMT2 genomic sequence, cDNA sequences available from the database, and alignment with related genes.

\section{Acknowledgments}

This work was supported by grants from the Searle Scholars Foundation (97-E-103), March of Dimes Birth Defects Foundation (FY00-418), and NIH (1R01GM61148) to J.B. L.B. was supported by NCI training grant 5-T32-CA09110.

The publication costs of this article were defrayed in part by payment of page charges. This article must therefore be hereby marked "advertisement" in accordance with 18 USC section 1734 solely to indicate this fact.

\section{References}

Bartee, L. and Bender, J. 2001. Two Arabidopsis methylation-deficiency mutations confer only partial effects on a methylated endogenous gene family. Nucleic Acids Res. 29: 2127-2134.

Bell, C.J. and Ecker, J.R. 1994. Assignment of 30 microsatellite loci to the linkage map of Arabidopsis. Genomics 19: 137-144.

Bender, J. and Fink, G.R. 1995. Epigenetic control of an endogenous gene family is revealed by a novel blue fluorescent mutant of Arabidopsis. Cell 83: 725-734.

Bevan, M. 1984. Binary Agrobacterium vectors for plant transformation. Nucleic Acids Res. 12: 8711-8721.

Clough, S.J. and Bent, A.F. 1998. Floral dip: A simplified method for Agrobacterium-mediated transformation of Arabidopsis thaliana. Plant J. 16: 735-743.

Colot, V. and Rossignol, J.-L. 1999. Eukaryotic DNA methylation as an evolutionary device. BioEssays 21: 402-411.

Finnegan, E.J. and Kovac, K.A. 2000. Plant DNA methyltransferases. Plant Mol. Biol. 43: 189-201.

Finnegan, E.J., Peacock, W.J., and Dennis, E.S. 1996. Reduced DNA methylation in Arabidopsis thaliana results in abnormal plant development. Proc. Nat1. Acad. Sci. 93: 8449-8454.

Frommer, M., McDonald, L.E., Millar, D.S., Collis, C.M., Watt, F., Grigg, G.W., Molloy, P.L., and Paul, C.L. 1992. A genomic sequencing protocol that yields a positive display of 5-methylcytosine residues in individual DNA strands. Proc. Natl. Acad. Sci. 89: 1827-1831.

Goyon, C., Nogueira, T.I., and Faugeron, G. 1994. Perpetuation of cytosine methylation in Ascobolus immersus implies a novel type of maintenance methylation. J. Mol. Biol. 240: 42-51.

Henikoff, S. and Comai, L. 1998. A DNA methyltransferase homolog with a chromodomain exists in multiple polymorphic forms in Arabidopsis. Genetics 149: 307-318.

Jeddeloh, J.A., Bender, J., and Richards, E.J. 1998. The DNA methylation locus DDM1 is required for maintenance of gene silencing in Arabidopsis. Genes \& Dev. 12: 1714-1725.

Jeddeloh, J.A., Stokes, T.L., and Richards, E.J. 1999. Maintenance of genomic methylation requires a SWI2/SNF2-like protein. Nat. Genet. 22: 94-97.

Kakutani, T., Jeddeloh, J.A., Flowers, S.K., Munakata, K., and Richards, E.J. 1996. Developmental abnormalities and epimutations associated with DNA hypomethylation mutations. Proc. Natl. Acad. Sci. 93: 12406-12411.

Konieczny, A. and Ausubel, F.M. 1993. A procedure for mapping Arabidopsis mutations using co-dominant ecotype-specific PCR-based markers. Plant J. 4: 403-410.

Li, E., Bestor, T.H., and Jaenisch, R. 1992. Targeted mutation of the DNA methyltransferase gene results in embryonic lethality. Cell 69: 915926.

Lindroth, A.M., Cao, X., Jackson, J.P., Zilberman, D., McCallum, C.M., Henikoff, S., and Jacobsen, S.E. 2001. Requirement of CHROMOMETHYLASE3 for maintenance of CpXpG methylation. Science 292: 2077-2080..

Luff, B., Pawlowski, L., and Bender, J. 1999. An inverted repeat triggers cytosine methylation of identical sequences in Arabidopsis. Mol. Cel1 3: 505-511. 
Bartee et al.

McCallum, C.M., Comai, L., Greene, E.A., and Henikoff, S. 2000. Targeted screening for induced mutations. Nat. Biotechnol. 18: 455-457.

Melquist, S., Luff, B., and Bender, J. 1999. Arabidopsis PAI gene arrangements, cytosine methylation and expression. Genetics 153: 401-413.

Niyogi, K.K., Last, R.L., Fink, G.R., and Keith, B. 1993. Suppressors of trp1 fluorescence identify a new Arabidopsis gene, TRP4, encoding the anthranilate synthase $\beta$ subunit. Plant Cell 5: 1011-1027.

Papa, C.M., Springer, N.M., Muszynski, M.G., Meeley, R., and Kaeppler, S.M. 2001. Maize chromomethylase Zea Methyltransferase2 is required for CpNpG methylation. Plant Cell (in press).

Ronemus, M.J., Galbiati, M., Ticknor, C., Chen, J., and Dellaporta, S.L. 1996. Demethylation-induced developmental pleiotropy in Arabidopsis. Science 273: 654-657.

Rose, T. M., Schultz, E.R., Henikoff, J.G., Pietrokovski, S., McCallum, C.M., and Henikoff, S. 1998. Consensus-degenerate hybrid oligonucleotide primers for amplification of distantly related sequences. Nucleic Acids Res. 26: 1628-1635.

Selker, E.U., Fritz, D.Y., and Singer, M.J. 1993. Dense nonsymmetrical DNA methylation resulting from repeat-induced point mutation in Neurospora. Science 262: 1724-1728.

Soppe, W.J.J., Jacobsen, S.E., Alonso-Blanco, C., Jackson, J.P., Kakutani, T., Koornneef, M., and Peeters, A.J.M. 2000. The late flowering phenotype of fwa mutants is caused by gain-of-function epigenetic alleles of a homeodomain gene. Mol. Cell 6: 791-802.

Vongs, A., Kakutani, T., Martienssen, R.A., and Richards, E.J. 1993. Arabidopsis thaliana DNA methylation mutants. Science 260: 19261928

Yoder, J.A., Walsh, C.P., and Bestor, T.H. 1997. Cytosine methylation and the ecology of intragenomic parasites. Trends Genet. 13: 335340. 


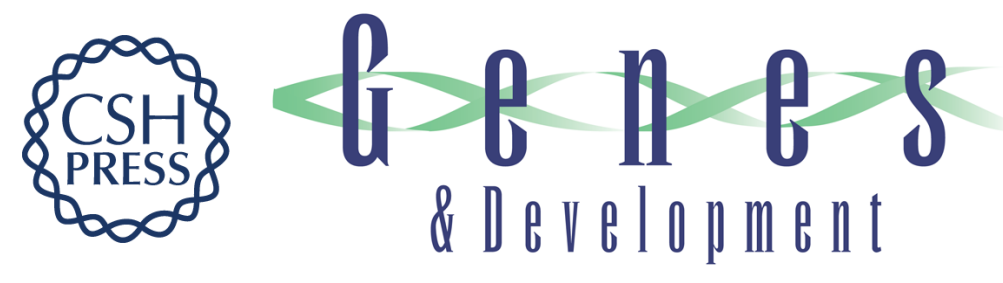

\section{Arabidopsis $\mathrm{cm} 33$ chromomethylase mutations block non-CG methylation and silencing of an endogenous gene}

Lisa Bartee, Fabienne Malagnac and Judith Bender

Genes Dev. 2001, 15:

Access the most recent version at doi:10.1101/gad.905701

References This article cites 27 articles, 11 of which can be accessed free at: http://genesdev.cshlp.org/content/15/14/1753.full.html\#ref-list-1

License

Email Alerting

Receive free email alerts when new articles cite this article - sign up in the box at the top Service right corner of the article or click here.

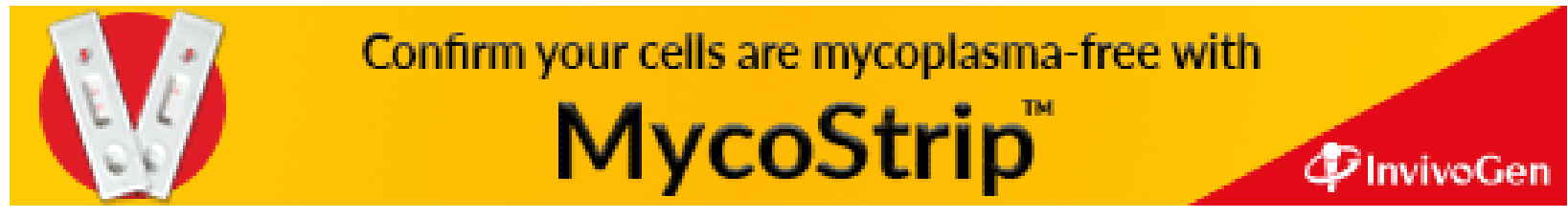

